\title{
Response of shock wave deformation in AA5086 aluminum alloy
}

\author{
Nachiketa Ray ${ }^{\mathrm{a}}$, Gopalan Jagadeesh ${ }^{\mathrm{b}}$, Satyam Suwas ${ }^{\mathrm{a}, *}$ \\ a Department of Materials Engineering, Indian Institute of Science, Bangalore 560012, India \\ ${ }^{\mathrm{b}}$ Department of Aerospace Engineering, Indian Institute of Science, Bangalore 560012, India
}

\section{A R T I C L E I N F O}

\section{Article history:}

Received 9 July 2014

Received in revised form

5 October 2014

Accepted 7 October 2014

Available online 23 October 2014

\section{Keywords:}

Rolling

Shock waves

Microstructure

Texture

Hardness

TEM

\begin{abstract}
A B S T R A C T
The AA5086 aluminum alloy sheets with different starting textures were subjected to shock wave deformation with an input impulse of $\sim 0.2$ Ns. Microstructural examination indicate no significant change in grain size; however, the evolution of substructure manifesting intra-granular misorientation was evident. The improvement in hardness indicates the absence of recovery and strain hardening during shock deformation. Shock deformed samples show characteristic texture evolution with high Brass $\{110\}<112\rangle$ component. The study demonstrates the viability of high velocity forming of AA5086 aluminum alloy sheet using shock wave.
\end{abstract}

(c) 2014 Elsevier B.V. All rights reserved.

\section{Introduction}

In contrast to conventional sheet metal forming processes, the techniques involving high velocity forming would serve as a "friction-less punch" to deform the thin sheets and would be much more effective in terms of energy transfer (K.E. $\propto v^{2}$ ). Although this idea is intriguing and the process is quite sophisticated, the evolution of microstructure and texture during such a process is likely to be different from conventional processing. High velocity forming using explosive gases, pulsed magnetic fields and electrical arc discharges are today being used in the metal forming industry for forming large components which would otherwise require unreasonably high pressures and inconceivable sized press. Explosive forming is rather ancient and goes way back to the nineteen hundreds when it was first used by Daniel Adamson, an English engineer known for his success in manufacturing boilers [1]. Table 1 gives us a general idea about the deformation velocities during sheet metal forming.

High velocity forming of thin sheets is one of the popular methods of obtaining shapes that are difficult to obtain from conventional metal forming [2,3]. Previously, attempts had been made to study the dynamic behavior of shock wave loaded plates using a conventional shock tube by Stoffel [4,5]. He used various visco-plastic models to simulate the deformation of the shockloaded plates and compared dynamically deformed plates vis-avis quasi-statically deformed plates in terms of mid-point

\footnotetext{
* Corresponding author.

E-mail address: satyamsuwas@materials.iisc.ernet.in (S. Suwas).
}

deflection, strain distribution and repetitive loads. However, this study did not reveal any micromechanism of deformation under shock loading. It is quite natural to assume that such process would influence the microstructural feature of materials, for example, substructural features and crystallographic texture. It is well known that these aspects dictate the properties and performance of the final product [6].

The response of such a forming process on the evolution of microstructure and crystallographic texture is not very clear till date. A few investigations have been made on changes in texture after shock wave loading earlier, however, in a more general manner. Different authors had contradicting views regarding changes in crystallographic orientation after shock wave loading. De Angelis and Cohen [7] observed texture changes in shock loaded copper. On the contrary Higgins [8] and Trueb [9] reported no such changes in texture of copper and nickel. Rose and Berger [10] were the first to report micro-structural changes after shock wave loading. Dhere et al. [11] observed texture changes in commercially pure aluminum and compared the changes in texture and sub-structural evolution between conventional cross-rolling and shock wave deformation.

In order to examine the effect of initial texture, if any, on the texture of shock deformed material, unidirectionally rolled and multi-step cross rolled samples were chosen. Such processing are known to generate different texture keeping the microstructural features almost similar [12-14]. In this prospect, the motivation for the present work is to explore the possibilities of using shock wave loading as a means to deform thin metal sheets and to investigate the changes in microstructure and texture. The material was 
chosen as a strain hardenable AA5086 aluminum alloy which is known to develop characteristic texture under different loading conditions [15-17].

\section{Experimental approach}

\subsection{Material and processing}

For the present investigation AA5086 aluminum alloy was used, the chemical composition of which is given in Table 2. The alloy was received in the form of $6 \mathrm{~mm}$ thick hot-rolled sheets. The as-received sheets were further processed by hot rolling $\left(400{ }^{\circ} \mathrm{C}\right)$ followed by cold rolling (room temperature) either in unidirectional manner (UDR) or multi-step cross rolling (MSCR). In multistep cross rolling, the sheets were rotated $90^{\circ}$ about the sheet normal after each pass. All the samples were reduced by $10 \%$ in each pass to obtain a final thickness of $0.5 \mathrm{~mm}$. The rolled sheets were annealed at $400{ }^{\circ} \mathrm{C}$ for $1 \mathrm{~h}$. Discs of $80 \mathrm{~mm}$ diameter were cut from each of the rolled plus annealed sheet.

The so-obtained discs were subjected to a high strain rate loading normal to their rolling plane in a conventional shock tube. A conventional shock tube is essentially a device in which a normal shock front is produced by sudden bursting of a diaphragm that separates a gas at high pressure from the one at lower pressure. The schematic of this setup is shown in Fig. 1. The driven section of the tube was evacuated to 0.3 bars whereas the driver section was filled with helium to build up a pressure enough to rupture the diaphragm. After the diaphragm ruptures, a compression wave is formed in the driven section, which rapidly steepens to form a shock front. The shock wave, as it travels along the lowpressure section, acts like a piston, continuously compressing the

Table 1

Deformation velocities for different forming techniques.

\begin{tabular}{llc}
\hline Forming Technique & Method & $\begin{array}{l}\text { Deformation velocity } \\
(\mathbf{m} / \mathbf{s})\end{array}$ \\
\hline Conventional forming & Hydraulic press & 0.06 \\
& Mechanical press & 1.5 \\
& Drop hammers & 6.1 \\
\multirow{2}{*}{ High velocity forming } & Low explosives/explosive gas & 150 \\
& High explosive & 300 \\
& Electro-hydraulic & 300 \\
& Electromagnetic & 300 \\
\hline
\end{tabular}

Table 2

Chemical composition (wt\%) of the AA5086 alloy used in present study.

\begin{tabular}{llllllllll}
\hline $\mathrm{Al}$ & $\mathrm{Mg}$ & $\mathrm{Mn}$ & $\mathrm{Cr}$ & $\mathrm{Cu}$ & $\mathrm{Fe}$ & $\mathrm{Si}$ & $\mathrm{Ti}$ & $\mathrm{Zn}$ & Other \\
\hline 93.36 & 4.0 & 1.06 & 0.02 & 0.01 & 0.65 & 0.37 & 0.28 & 0.2 & 0.05 \\
\hline
\end{tabular}

gas in the tube. Simultaneously, an expansion wave moves back into the driver section. It is the shock front which deforms the exposed sheet while it is constrained along its circular edge. This tends to the formation of a spherical cap from a flat disc as shown in Fig. 2.

The diaphragm used was a commercially pure aluminum disc with an induced fracture plane (a cross indent with constant depth). Thus the pressure in the driver section could be varied by manipulating the thickness of the diaphragm and depth of the indent. Three specific diaphragms $\left(2^{1 / 5}, 2^{1 / 3}\right.$ and $\left.1.6^{1 / 3}\right)$ were used for maintaining three different pressure levels in the driver section. In the notation, the exponent denotes the fraction of the thickness dented out and the base indicates the thickness of the diaphragm in millimeters. Two piezoelectric pressure transducers (Sensor 1 and Sensor 2) were used to measure the pressure values and shock speed while the third transducer (Sensor 3) was flush mounted at the end flange to calculate the impulse felt by the sample. For this case the sensor was fixed in place of the sample to feel the peak over pressure. Fig. 1 illustrates the respective position of the sensors.

\subsection{Strain measurements}

The shock loaded sheets experience three principal strains namely circumferential strain $\left(\varepsilon_{\theta}\right)$, radial strain $\left(\varepsilon_{r}\right)$, and thickness strain $\left(\varepsilon_{\mathrm{t}}\right)$. Fig. 3 shows a schematic of the deformed and the initial sample and also illustrates the principal strain directions.

The effective strain can be calculated [18] as

$\bar{\varepsilon}=\frac{1}{1+\nu^{\prime}} \sqrt{\frac{1}{2}\left[\left(\varepsilon_{\theta}-\varepsilon_{r}\right)^{2}+\left(\varepsilon_{\theta}-\varepsilon_{t}\right)^{2}+\left(\varepsilon_{t}-\varepsilon_{r}\right)^{2}\right]}$

where $\nu^{\prime}$ is the effective Poisson's ratio which has a value of 0.5 for plastic, creep and hyper elastic strains. Thus the effective strain

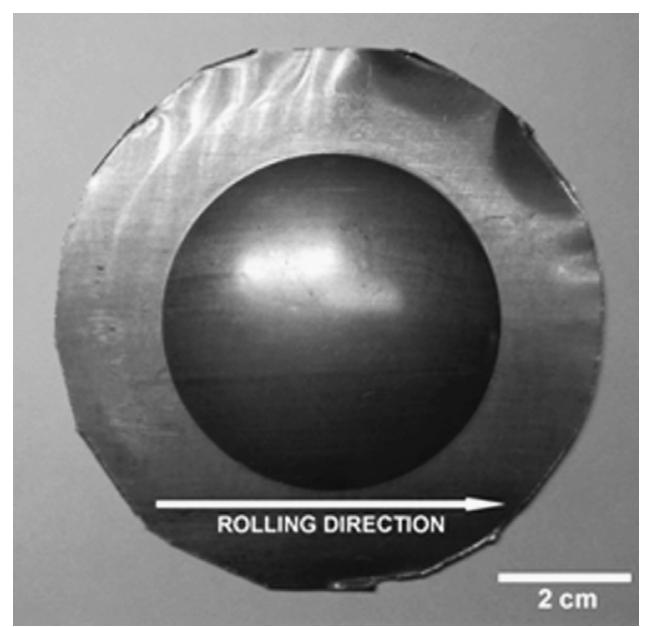

Fig. 2. Unidirectional and cold rolled sheet deformed in conventional shock tube.

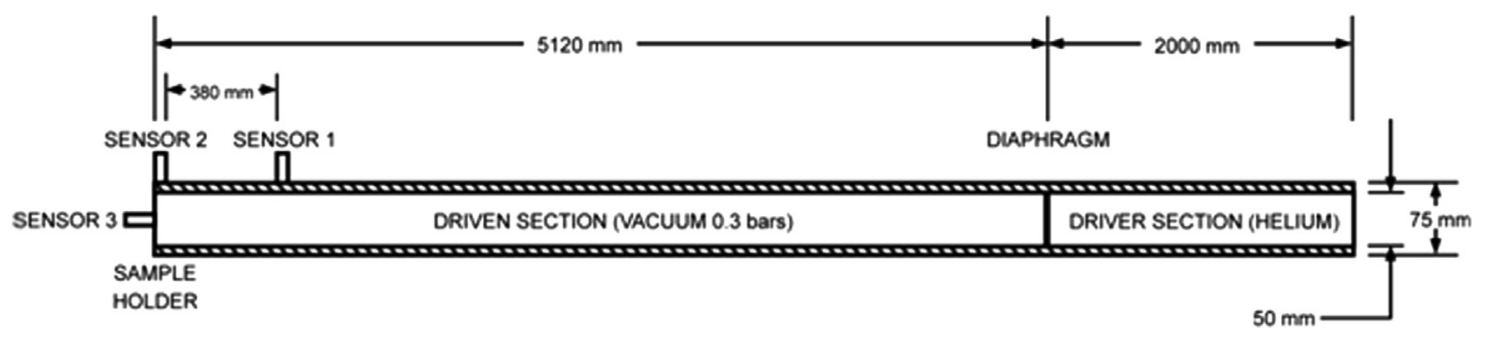

Fig. 1. Schematic layout of the conventional shock tube. 


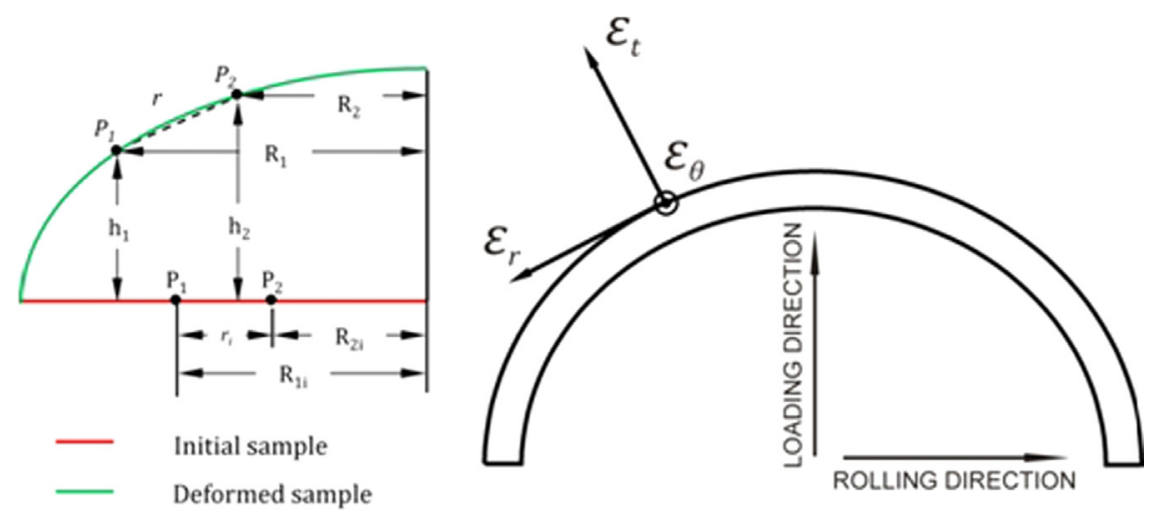

Fig. 3. Schematic representation of measurement of circumferential and radial strains.

reduces to

$\bar{\varepsilon}=\sqrt{\frac{2}{9}\left[\left(\varepsilon_{\theta}-\varepsilon_{r}\right)^{2}+\left(\varepsilon_{\theta}-\varepsilon_{t}\right)^{2}+\left(\varepsilon_{t}-\varepsilon_{r}\right)^{2}\right]}$

Since the deformed plate shows a balanced biaxial condition of strain,

$\varepsilon_{\theta}=\varepsilon_{r}=-\frac{\varepsilon_{t}}{2}$

After substituting Eq. (3) in Eq. (2) the final expression for effective strain becomes

$\bar{\varepsilon}=\sqrt{\varepsilon_{t}^{2}}$

Thus we only need to measure thickness strain $\left(\varepsilon_{t}\right)$ to estimate the effective strain. For a sheet of initial thickness $\left(t_{0}\right)$ and initial area of exposure $\left(A_{0}\right)$, which after deformation has thickness, $t$ and deformed area, $A$,thickness strain can be calculated from the following expression assuming volume constancy,

$\varepsilon_{t}=\ln \frac{t}{t_{0}}=\ln \frac{A_{0}}{A}$

Assuming the geometry presented in Fig. 4, thickness strain $\left(\varepsilon_{\mathrm{t}}\right)$ from Eq. (5) becomes

$\varepsilon_{t}=\ln \frac{\pi r^{2}}{2 \pi \rho h}=\ln \frac{r^{2}}{2 \rho h}$

where

$\rho=\frac{r^{2}+h^{2}}{2 h}$

It is evident from Eq. (6) that the mid-point deflection $(h)$ was enough to calculate the strain in the material. Thus after the 'cap' was sectioned along the center of the disc the mid-point displacement was measured using a height gauge.

\subsection{Microstructural and texture characterization}

The deformed samples were sectioned along the rolling direction using Electro-Discharge Machining (EDM) and further characterized for microstructure and texture. Electron back-scattered diffraction (EBSD) studies were done on the transverse plane of the samples while bulk X-ray texture measurement as well as transmission electron microscopy was carried out on the rolling plane.

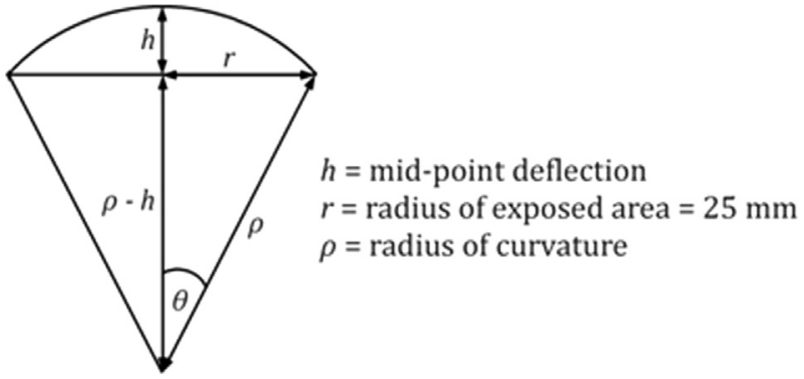

Fig. 4. Nomenclature used for the calculation of thickness strain.

For EBSD measurements, the transverse surface was carefully polished using conventional metallography followed by electropolishing ${ }^{1}$ using an electrolyte consisting $78 \mathrm{ml}$ perchloric acid, $90 \mathrm{ml}$ distilled water, $730 \mathrm{ml}$ ethanol and $100 \mathrm{ml}$ butoxyethanol. EBSD for all the samples was performed using a scanning electron microscope $(\mathrm{SEM})^{2}$ with an accelerating voltage of $25 \mathrm{kV}$. The step size for the EBSD scans was varied from sample to sample but was kept within the range of $0.7-1.5 \mu \mathrm{m}$. The scanned area was kept sufficiently large to include at least 3000 grains. The orientation data obtained from EBSD scan was analyzed using OIM ${ }^{\mathrm{TM}}$ Analysis Software. ${ }^{3}$

For the bulk texture measurement, the rolling plane of the sheet was polished and investigated in a X-ray texture goniometer based on Schulz reflection geometry ${ }^{4}$ using $\mathrm{Cu} \mathrm{K}_{\alpha}$ radiation. Four incomplete pole figures $\{111\},\{200\},\{220\},\{311\}$ were measured experimentally (The scan was done till $\psi=75^{\circ}$ ). Quantitative texture analysis was carried out for calculating the orientation distribution functions (ODF) using LaboTex ${ }^{5}$ software. Arbitrarily Defined Cells (ADC) method was used for the calculation of ODF and orthorhombic symmetry was imposed.

The samples for TEM were grinded and polished up to $100 \mu \mathrm{m}$ using conventional metallography followed by electro-polishing ${ }^{6}$ in an electrolyte containing 30\% nitric acid and 70\% methanol. The polishing was done at $17 \mathrm{~V}$ and $40 \mathrm{~mA}$ and the temperature was maintained at $-25^{\circ} \mathrm{C}$. Bright field images were captured using conventional transmission electron microscopy ${ }^{7}$ at an accelerating voltage of $200 \mathrm{kV}$.

\footnotetext{
${ }^{1}$ Struers ${ }^{\circledR}$ Lectropol-5, Struers A/S, Germany.

2 QUANTA 200, FEI, The Netherlands.

3 EDAX Inc., USA.

${ }^{4}$ D8 Discover with GADDs, Bruker AXS, Germany.

${ }^{5}$ LaboTex, LaboSofts.c., Poland.

${ }^{6}$ Fischione Instruments Twin-Jet Electropolisher.

7 JEOL 2000FX.
} 


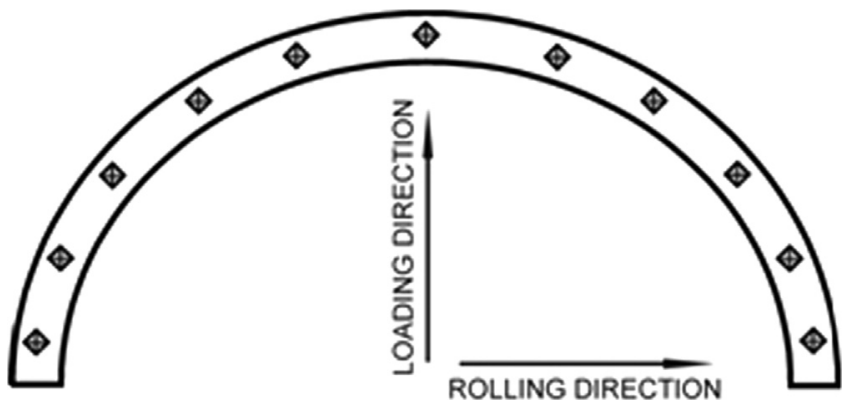

Fig. 5. Transverse section showing the position of the indents for hardness measurements.

\subsection{Micro-hardness tests}

The hardness of the deformed samples was measured on the transverse section by employing the Vickers hardness technique. The sectioned samples were mounted on an epoxy based resin but it was ensured that the bottom of the transverse surface should protrude out. By doing this the hardness of the mount would not come into play during measurement. Indents were made along the thickness of the deformed sheet as shown in Fig. 5.

\section{Results and discussion}

\subsection{Impulse measurement}

Three different diaphragms were used to generate shock waves, as mentioned earlier and to estimate the pressure felt by the samples, the rolled sheet was replaced by Sensor 3. The impact pressure recorded from sensor 3 was plotted against time for all cases and is illustrated in Fig. 6. The impulse values were calculated by measuring the area under the constant pressure step (Table 3). The impulse experienced by the samples was the best way to define the loading conditions. The step loading of a shock front makes it very different from other kinds of high velocity loading such as a blast wave, where the pressure decreases exponentially after reaching the peak over pressure.

The macroscopic strain accumulated clearly indicates the degree of deformation undergone by the spherical cap. The effective strains in the spherical caps were calculated from Eqs. (4) and (6) and are presented in Table 4 for different impact pressures. This result clearly indicates that the macroscopic strain is not substantial (7-10\%). However such a dynamic deformation leads to significant modification in texture and microstructure.

\subsection{Microstructural evolution}

\subsubsection{Initial cold rolled and annealed sheet}

Microstructure of the initial $0.5 \mathrm{~mm}$ sheets (both UDR and MSCR) show mostly equiaxed grains ( $\sim 20 \mu \mathrm{m}$ diameter) on the transverse plane. This was due to the fact that the sheets were annealed after rolling. The microstructure of the annealed UDR and MSCR materials are shown in Fig. 7.

\subsubsection{Shock deformed cold-rolled and annealed UDR sheet}

The EBSD generated micrographs (also known as IPF maps) for the initial material and deformed sheets are shown in Fig. 8(a-c) where the black lines indicate high angle grain boundaries (misorientation $>15^{\circ}$ ) and the differently colored regions depict different grain orientation. Fig. 9(a) presents grain size distribution while Fig. 9(b) displays the grain boundary character distribution for these materials after deformation. Both the grain size and grain

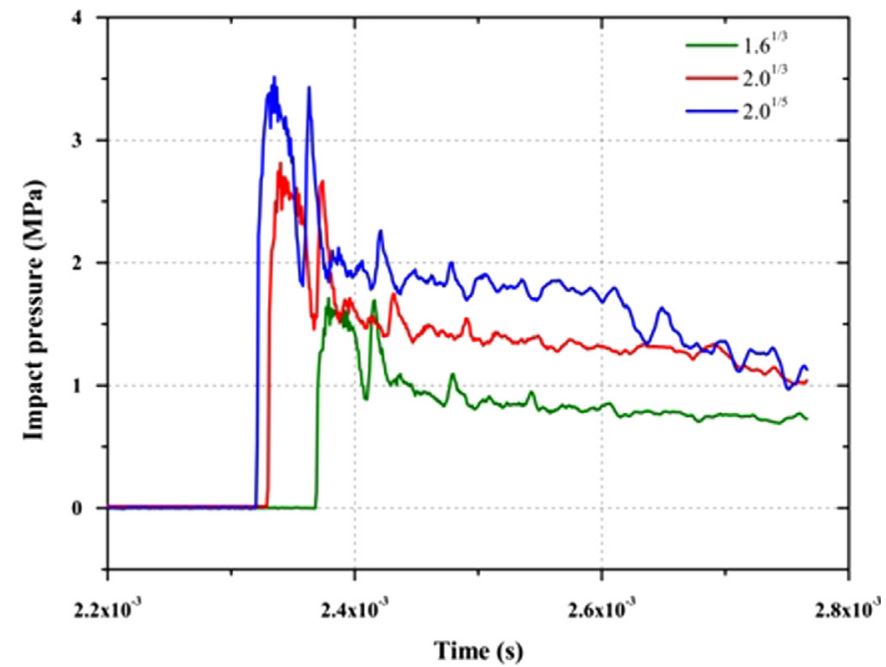

Fig. 6. Impact pressure values obtained from different diaphragms.

Table 3

Different pressure levels for each diaphragm.

\begin{tabular}{lll}
\hline Diaphragm used & Peak impact pressure $(P)(\mathrm{MPa})$ & Impulse $(I)(\mathrm{Ns})$ \\
\hline $1.6^{1 / 3}$ & 1.72 & 0.17 \\
$2.0^{1 / 3}$ & 2.82 & 0.28 \\
$2.0^{1 / 5}$ & 3.52 & 0.35 \\
\hline
\end{tabular}

Table 4

Effective strain measured for the deformed samples.

\begin{tabular}{lllll}
\hline Material condition & $\mathrm{P}(\mathrm{MPa})$ & $h(\mathrm{~mm})$ & $\rho(\mathrm{mm})$ & $\bar{\varepsilon}$ \\
\hline Hot rolling & & & & \\
$\quad$ MSCR & 3.52 & 6.02 & 54.92 & 0.056 \\
$\quad$ UDR & 2.82 & 6.83 & 49.17 & 0.072 \\
& 1.72 & 7.06 & 47.79 & 0.077 \\
& & & & \\
Cold rolling & & & & \\
$\quad$ MSCR & 3.52 & 8.80 & 39.91 & 0.117 \\
$\quad$ UDR & 3.52 & 7.81 & 43.92 & 0.093 \\
& 2.82 & 6.92 & 48.62 & 0.074 \\
\hline
\end{tabular}

boundary character distributions show only a little change after deformation. However, the orientation gradients within a grain were mapped to determine the extent of deformation in terms of grain orientation spread (GOS). The orientation of each pixel ( $g$ ) within a grain was measured with respect to the sample coordinate system and the average orientation was thus calculated. Finally the deviation of the orientation of each pixel from the average orientation within a grain was estimated as the grain orientation spread (GOS). In order to amplify the effect of orientation gradient, GOS maps were superimposed on the Image Quality (IQ) maps and are shown in Fig. 10(a-c). The figures clearly depict that the misorientation in terms of orientation spread increases as the amount of shock deformation imparted to the material increases.

\subsubsection{Shock deformed cold-rolled and annealed MSCR sheet}

The characterizations of MSCR plus annealed sheets were carried out in the same manner as done for the shock deformed unidirectional rolled sheets. The IPF and the GOS map of the deformed MSCR sample are shown in Figs. 8(d) and 10 (d) respectively. The IPF shows recrystallized grains in multi-step 

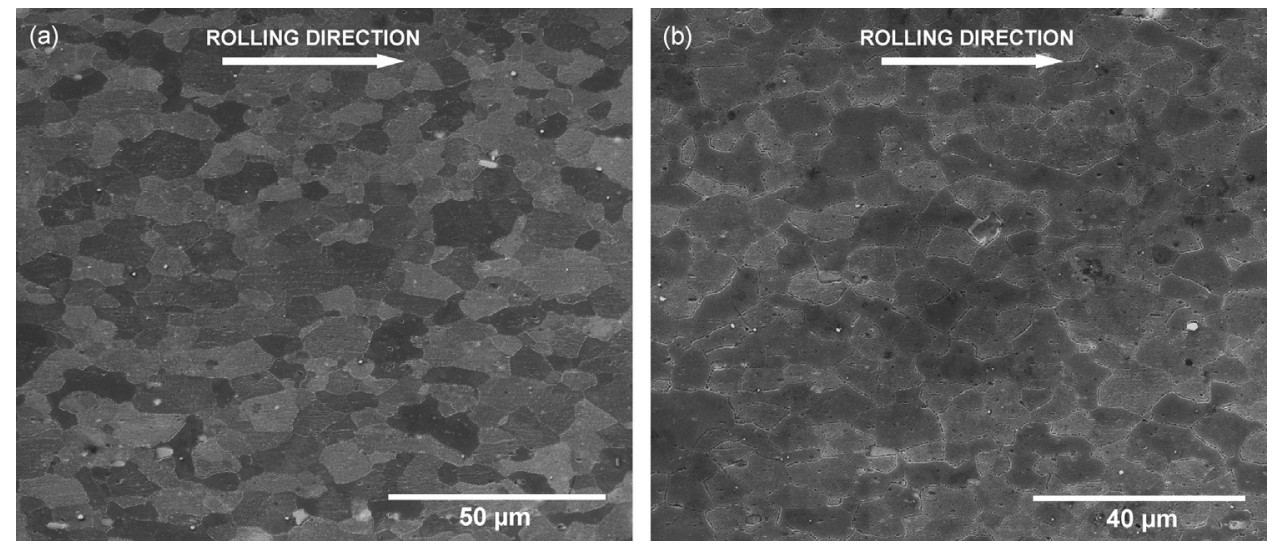

Fig. 7. SEM micrograph showing the transverse section of annealed AA5086 sheets processed by (a) UDR and (b) MSCR.

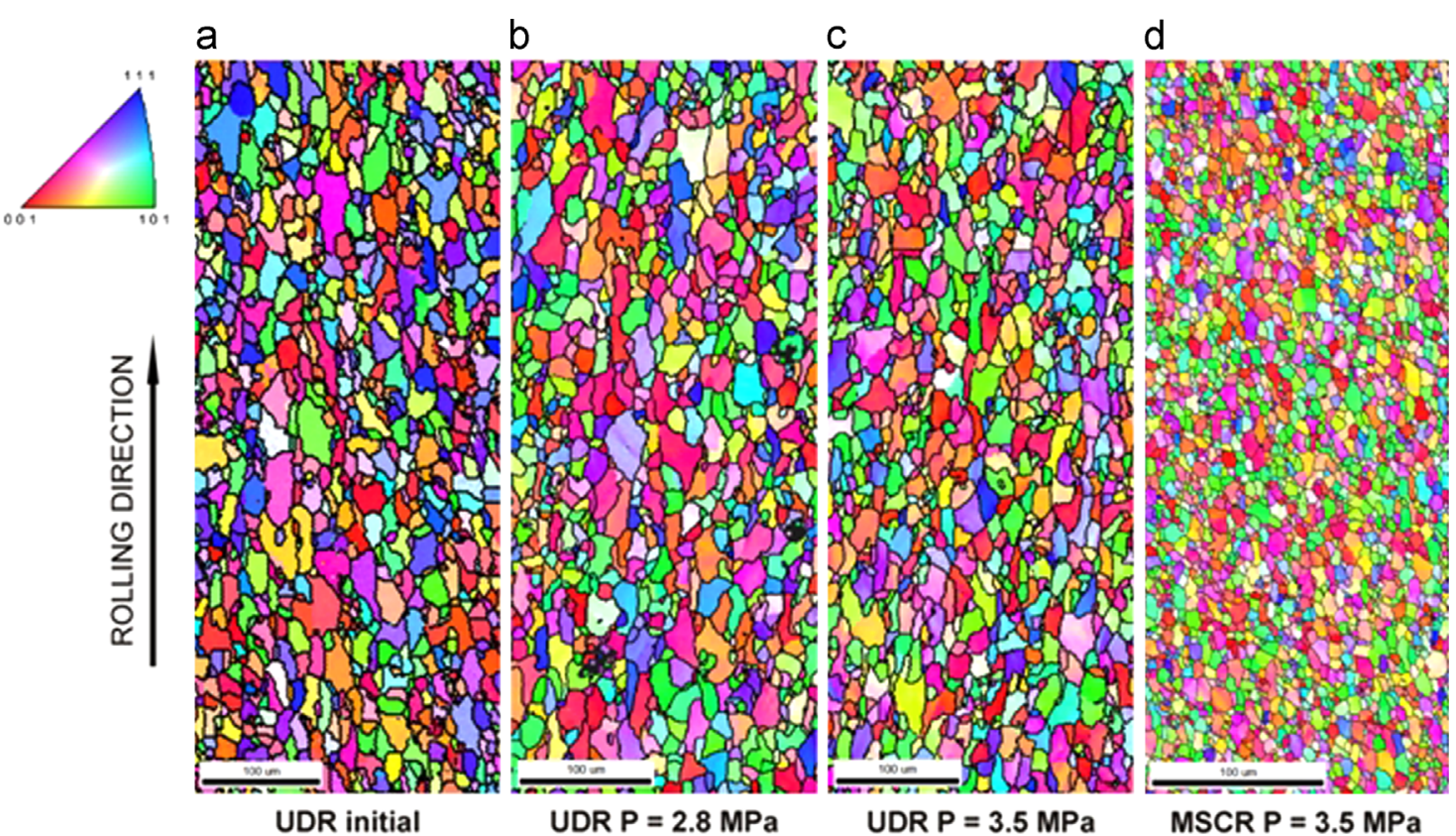

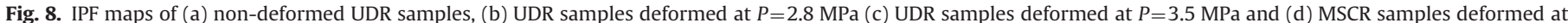
$P=3.5 \mathrm{MPa}$.
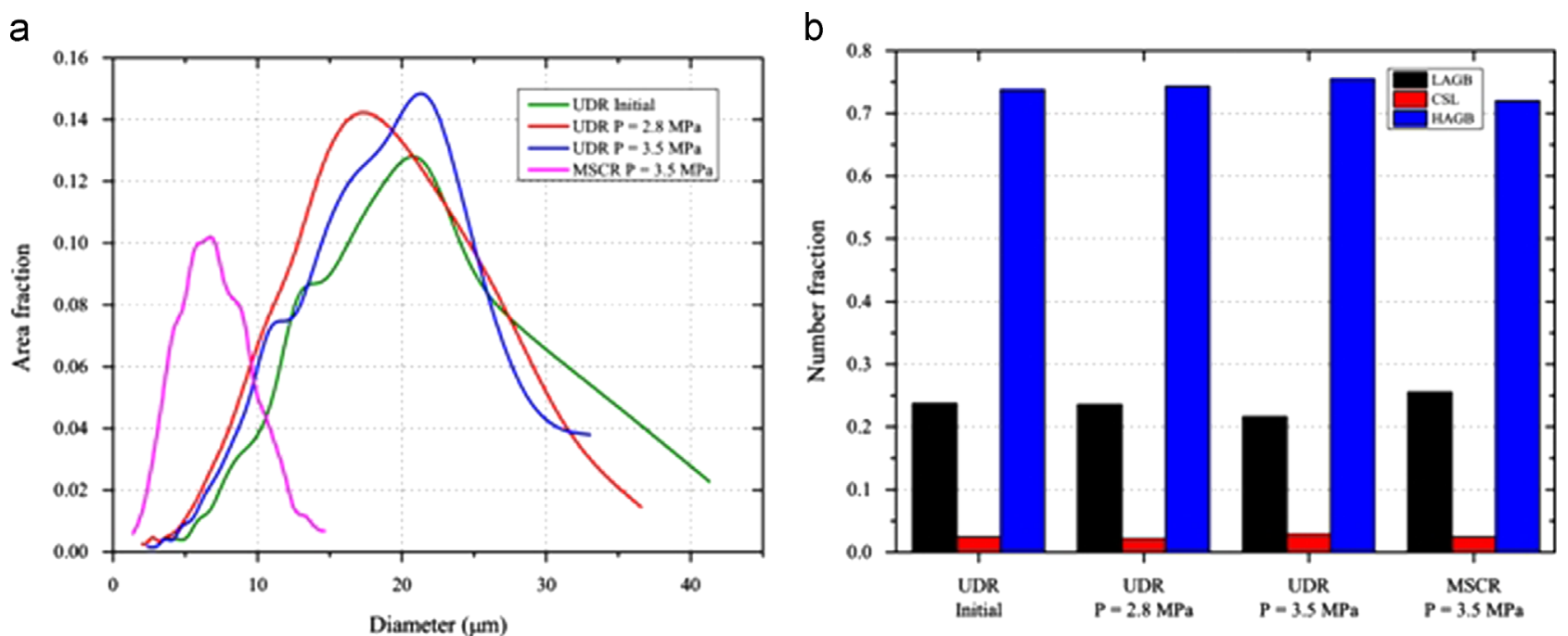

Fig. 9. (a) Grain size distribution and (b) Grain boundary character distribution at different impact pressures for UDR and MSCR samples. 


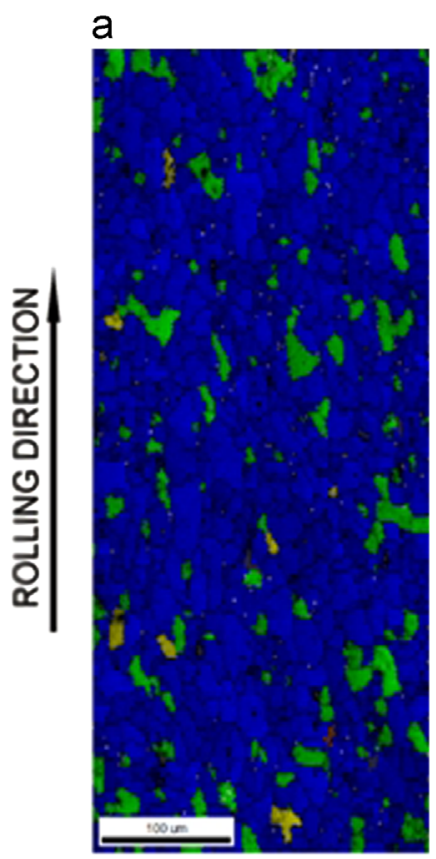

UDR initial b

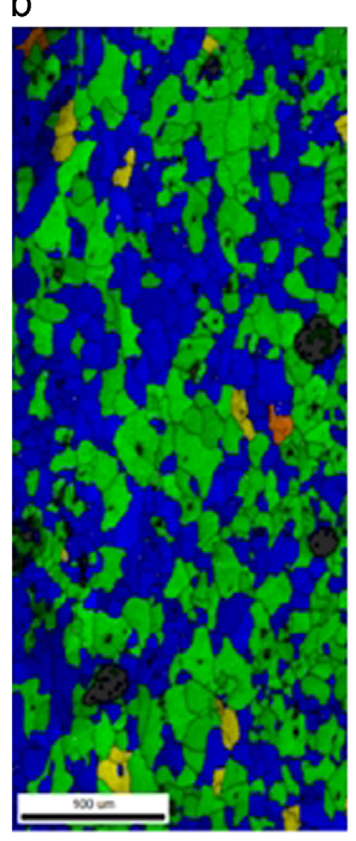

UDR $\mathrm{P}=2.8 \mathrm{MPa}$
C

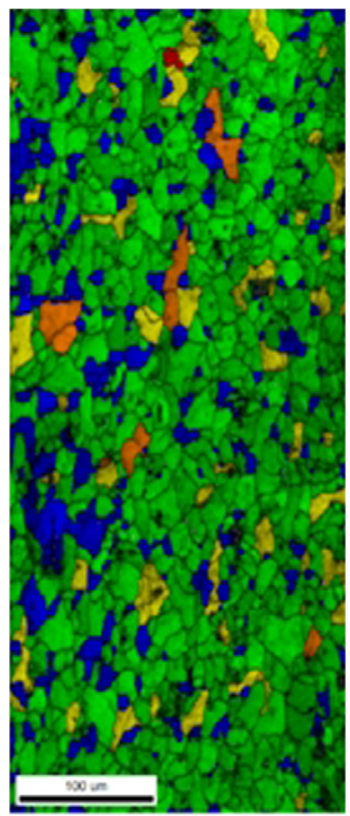

UDR $\mathrm{P}=3.5 \mathrm{MPa}$ d

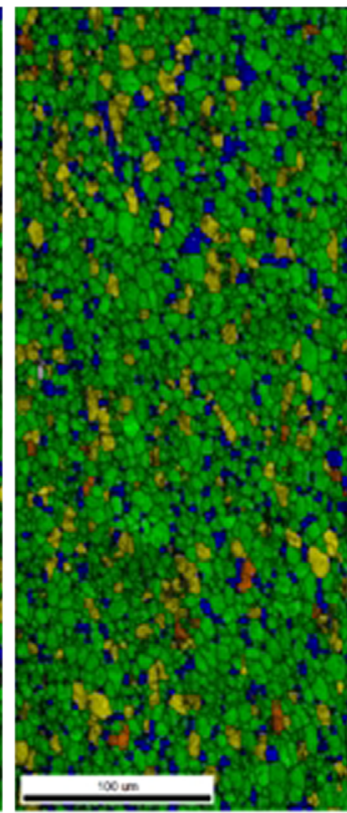

MSCR P $=3.5 \mathrm{MPa}$

\begin{tabular}{|c|c|c|c|c|c|}
\hline \multirow{3}{*}{$\begin{array}{c}\text { ORIENTATION } \\
\text { SPREAD }\left({ }^{\circ}\right)\end{array}$} & \multicolumn{4}{|c|}{ TOTALAREA FRACTION } \\
\cline { 3 - 6 } & & $\mathrm{P}=0$ & $\mathrm{P}=2.8 \mathrm{MPa}$ & $\mathrm{P}=3.5 \mathrm{MPa}$ & $\mathrm{P}=3.5 \mathrm{MPa}$ \\
\cline { 2 - 5 } & $0-1$ & 0.876 & 0.474 & 0.14 & 0.111 \\
\hline & $1-2$ & 0.107 & 0.491 & 0.753 & 0.734 \\
\hline & $2-3$ & 0.008 & 0.022 & 0.079 & 0.138 \\
\hline & $3-4$ & 0.000 & 0.003 & 0.022 & 0.010 \\
\hline & $4-5$ & 0.000 & 0.000 & 0.001 & 0.000 \\
\hline
\end{tabular}

Fig. 10. Grain Orientation Spread (GOS) maps imposed on the Image Quality (IQ) maps of (a) non-deformed UDR samples, (b) UDR samples deformed at $P=2.8$ MPa (c) UDR samples deformed at $P=3.5 \mathrm{MPa}$ and (d) MSCR samples deformed at $P=3.5 \mathrm{MPa}$.
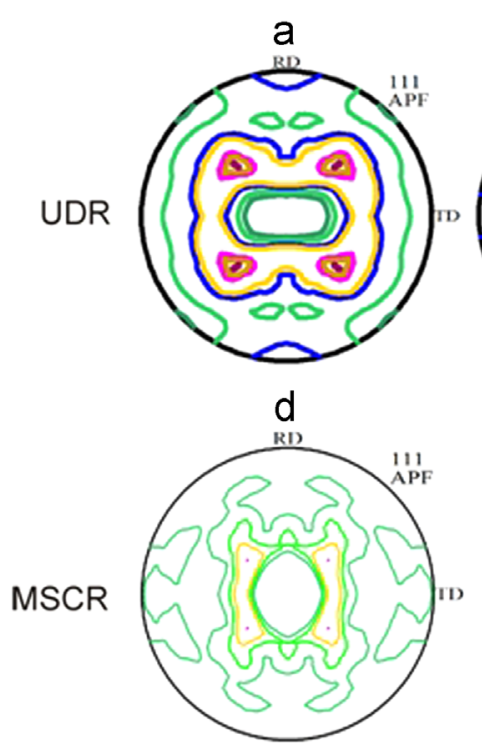

Initial
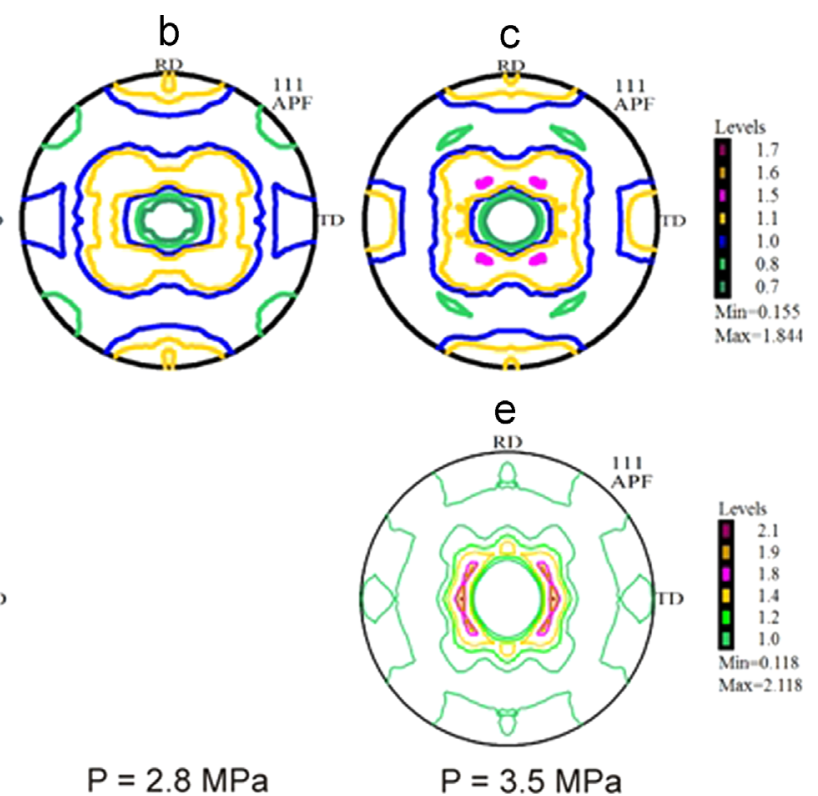

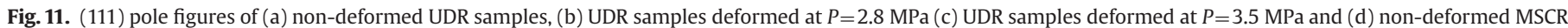
samples (e) MSCR samples deformed at $P=3.5 \mathrm{MPa}$. 

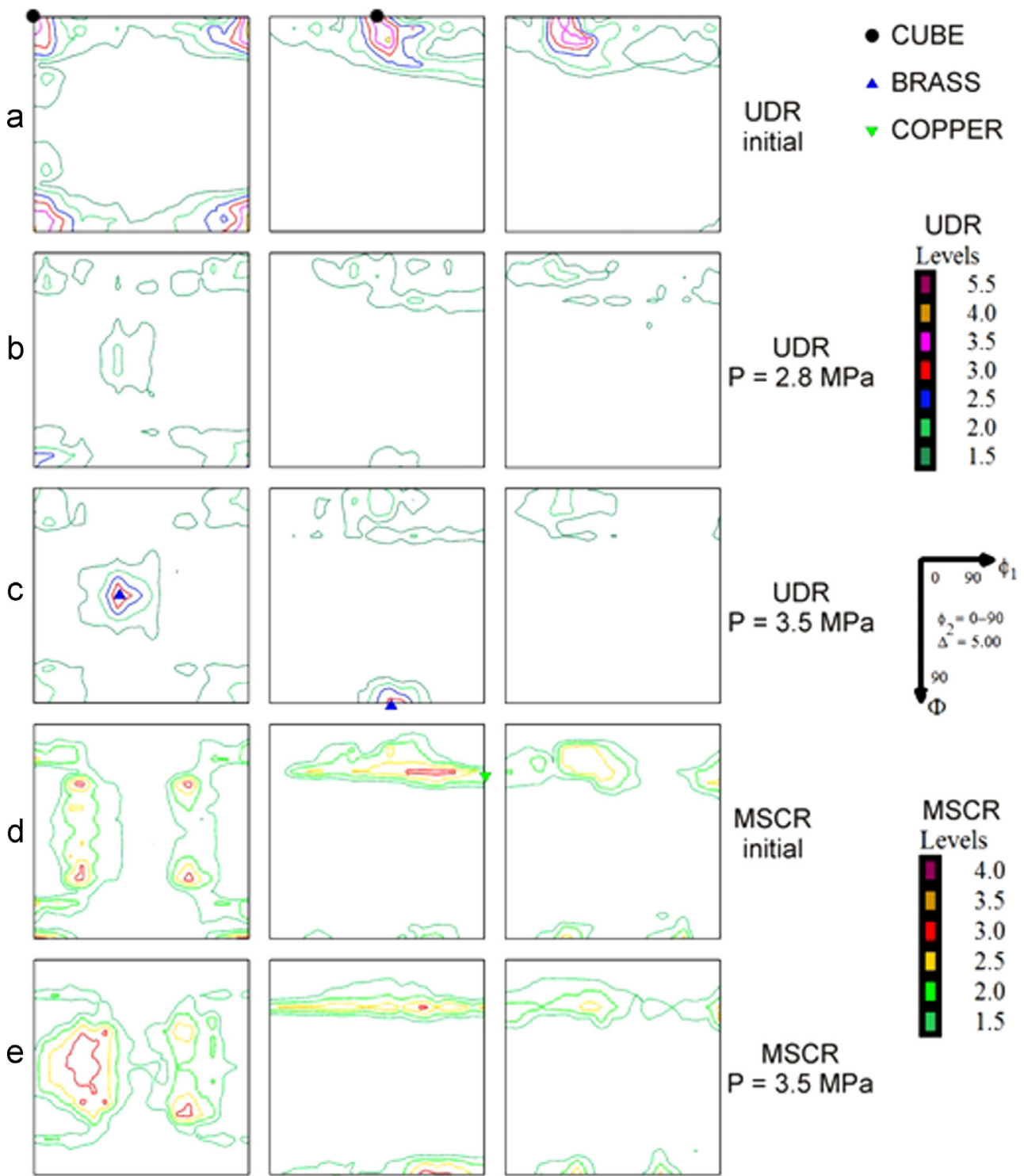

MSCR

Levels

4.0

- 3.5

3.0

2.5

2.0

MSCR

$\mathrm{P}=3.5 \mathrm{MPa}$

Fig. 12. Selected ODF sections of (a) non-deformed UDR samples, (b) UDR samples deformed at $P=2.8 \mathrm{MPa}$ (c) UDR samples deformed at $P=3.5 \mathrm{MPa}$ and (d) non-deformed MSCR samples (e) MSCR samples deformed at $P=3.5 \mathrm{MPa}$.

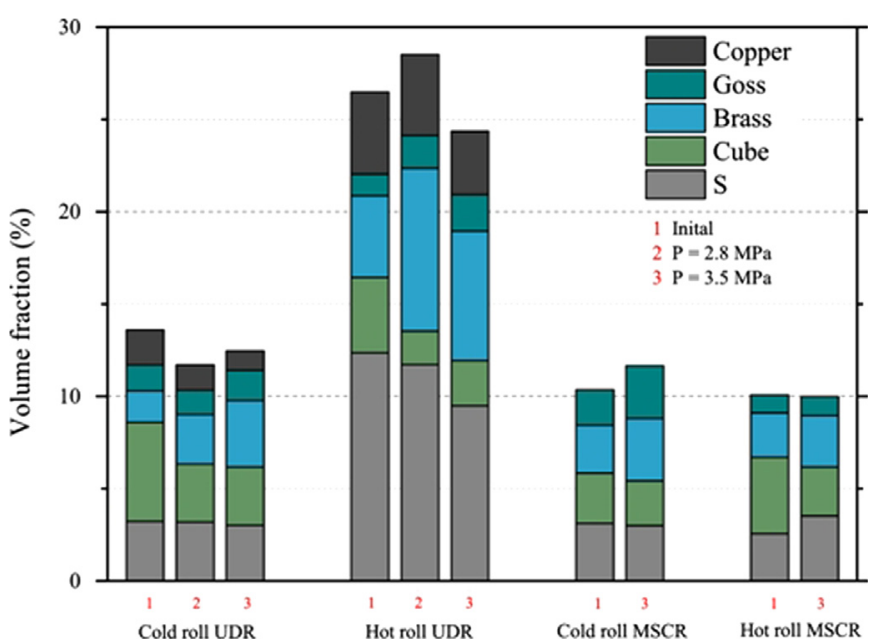

Fig. 13. Volume fraction of typical FCC texture components at different conditions for UDR and MSCR samples.

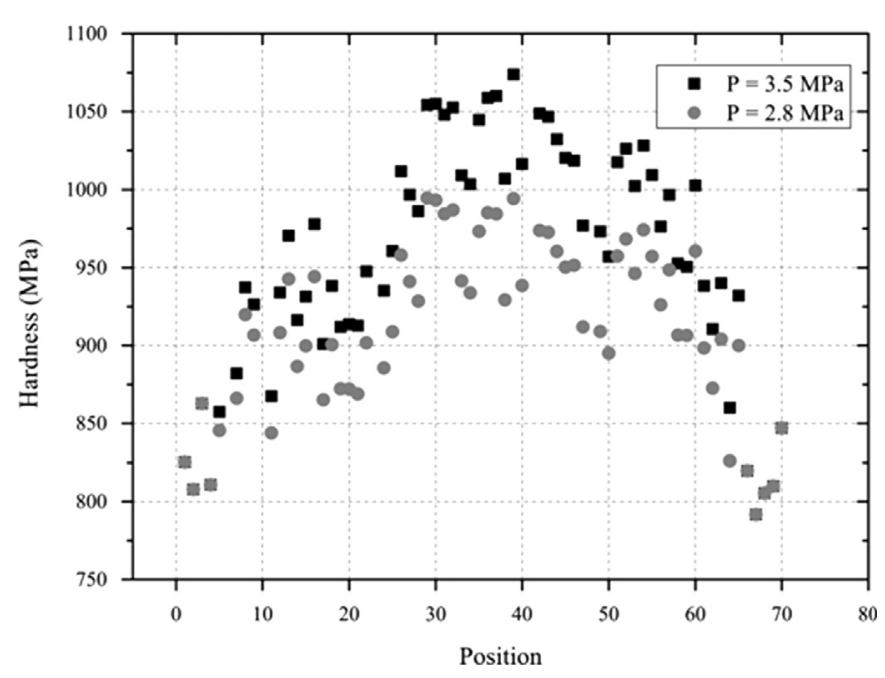

Fig. 14. Variation of hardness along the thickness of the deformed UDR samples. 
cross rolled samples, however, the GOS map clearly indicates the presence of strain in the microstructure in terms of grain rotation. The average grain size of the deformed sheet was around $7 \mu \mathrm{m}$ and the grain boundary character distribution was very similar to the UDR sheets.

\subsection{Texture evolution}

\subsubsection{Shock deformed cold-rolled and annealed UDR sheet}

Textural changes were monitored by measuring the pole figures using X-ray diffraction. Fig. 11(a-c) displays the (111) pole figures for the shock deformed UDR sheets. On comparing with texture of initial sample changes in texture are quite apparent. The (111) pole figures clearly indicate that the cube component $\{001\}$ $\langle 100\rangle$ has a sharp intensity for the initial material but diffuses gradually with increasing strain. The brass component $\{110\}\langle 112\rangle$ grows at the expense of the stable cube component. In order to carry out a detailed analysis of texture Orientation Distribution Function (ODF) was calculated.

Fig. $12(\mathrm{a}-\mathrm{c})$ shows the relevant $\varphi_{2}$ sections $\left(\varphi_{2}=0^{\circ}, 45^{\circ}\right.$ and $\left.65^{\circ}\right)$ as these section mostly describe the deformation texture component for FCC materials. By examining the intensity levels it is easy to conclude that the texture of the sample gets relatively weakened. Cube component literally disappeared and brass component strengthened with increasing strain.

\subsubsection{Shock deformed cold-rolled and annealed MSCR sheet}

Similar pole figure measurements were carried out for the shock deformed MSCR sheets. The (111) pole figures of the initial and shock deformed sheets are shown in Fig. 11(d) and (e) respectively. Fig. 12(d) and (e) shows the relevant $\varphi_{2}$ sections of the ODF for the initial and shock deformed sheet. Clear changes in texture can be observed yet the changes are not as drastic as in

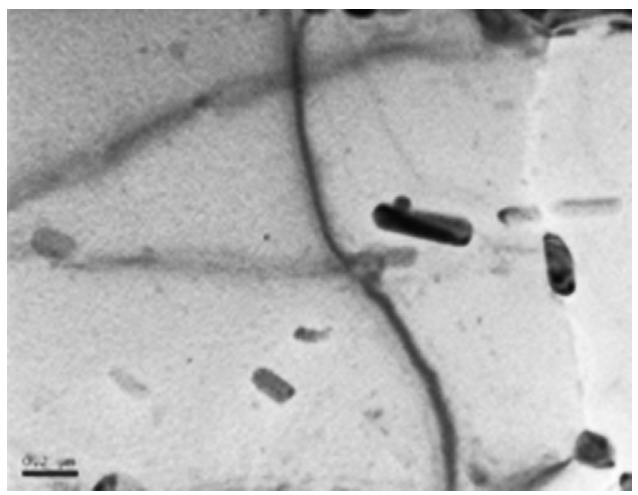

Fig. 15. TEM bright field image of the initial cold rolled and annealed sheet. the UDR processed samples. The initial texture of the sample itself is very weak and there seems to be a slight strengthening.

\subsubsection{Quantitative analysis}

By far a qualitative impression was conceived, but to determine the actual fraction of texture components it is desirable to calculate the volume fraction of each component in the orientation space. Let us consider a small volume element $\Delta \varphi_{1} \cdot \Delta \Phi \cdot \Delta \varphi_{2}$ which when integrated in all three directions gives the total volume of each texture component in the orientation space. Thus the volume elements having the Euler angles close to the ideal values (Fig. 12) correspond to respective texture component.

For such a quantitative analysis all the shock deformed samples were chosen to finally have a very clear overview, viz. cold and hot rolled, UDR and MSCR. These volume fractions were tabulated for the typical texture components in FCC and are illustrated in Fig. 13. The values shown in the figure are absolute and there was no comparison between the different initial rolling conditons. There were four different rolling conditons and each of them were subjected to shock wave loading at different pressure levels. From the figure it was quite evident that the brass component (deformation) grows at the expense of the cube component (recystallization). In general the cube component was very stable after annealing and an extensive deformation (eg. deformation banding) was required to form bass texture component which is sometimes also associated with deformation twinning (during the formation of brass texture).

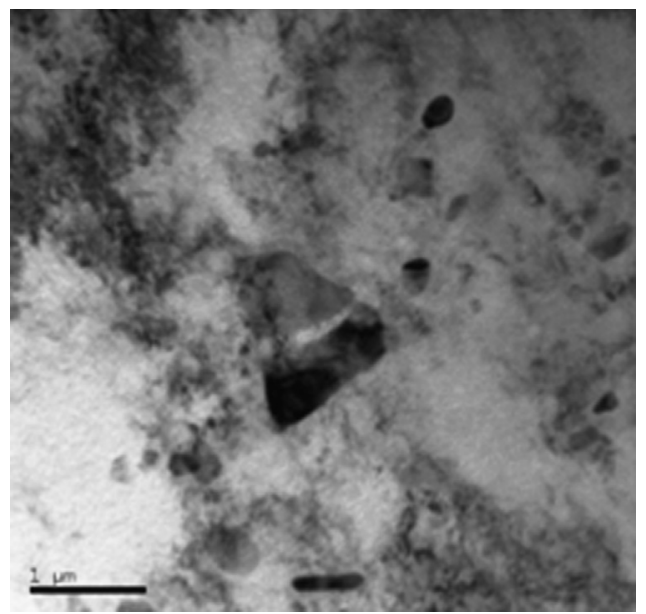

Fig. 17. TEM bright field image illustrating the fragmentation of dispersions.
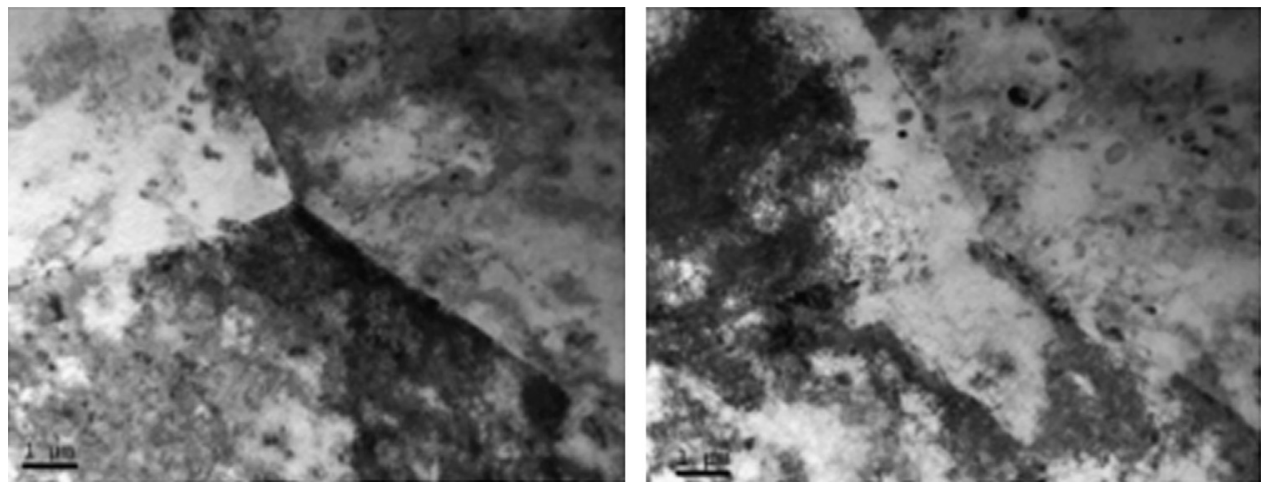

Fig. 16. TEM bright field image of the deformed sample from the same region at different tilt. 


\subsection{Hardness measurements}

The Vickers hardness of the deformed unidirectional cold rolled and annealed samples was measured and is illustrated in Fig. 14. The data points at $x=0$ and 70 represent the undeformed region of the spherical cap and hence the initial sample. A substantial increase in hardness in the mid region is evident. For the sample deformed at 3.5 MPa, the increase in hardness was about $273 \mathrm{MPa}$ and at 2.8 MPa impact pressure the increase was $194 \mathrm{MPa}$. These values also correspond to the increase in yield strength $\left(\sigma_{\mathrm{y}}\right)$. Thus we can conclude that shock waves at these pressure levels were enough to strain harden the material. This could be attributed due to dislocation interactions, which nucleate at the shock front due to the high deviatoric stresses produced by the shock wave. To confirm the presence and interaction of dislocations, TEM study was carried out for these samples.

\subsection{TEM studies}

Both the initial and the deformed materials were examined under transmission electron microscope. Since the starting material was in annealed condition no dislocation was observed even after tilting (Fig. 15). This was treated as a reference sample mainly to study the distinguished features after shock wave loading. The figure clearly depicts the grain boundary and morphology of dispersion.

The dislocation substructure observed in the deformed sample $(P=3.5 \mathrm{MPa})$ was similar to that observed by Rose and Berger [10]. A series of low magnification bright-field TEM micrographs were taken from the same area in the matrix (a triple junction) at different specimen tilt conditions to qualitatively construe the dislocation density (Fig. 16). This essentially confirms the formation of dislocation substructure after shock loading. No sign of recovery or cell formation by dislocation rearrangement was observed after shock loading. This could be due to the fact that the dislocations were uniformly generated at the shock front and lag behind. Another salient observation made during the TEM study was the fragmentation of the dispersions after shock wave loading, which is shown in Fig. 17.

\section{Conclusions}

Experiments were carried out to explore the possibility of forming of thin AA5086 aluminum alloy sheets using shock waves. The analysis of microstructure, bulk texture and hardness were carried out for the initial as well as for the shock wave deformed materials. Based on the experimental results the following conclusions can be drawn:
1. It is possible to use shock loading as a forming operation for thin AA5086 aluminum alloy sheets by using very small magnitude of impulse ( $\sim 0.2 \mathrm{Ns})$, which is negligible in conventional forming techniques.

2. Although no significant change in grain size, morphology and grain size distribution occurs after shock wave loading, there is substructural evolution in the material, which manifests as intra-granular misorientation. Substructural features as seen in TEM micrographs indicate that the material undergoes plastic deformation after shock wave loading. The occurrence of dislocations with no sub-grain formation suggests minimal recovery during deformation.

3. Appreciable changes in bulk texture are observed, however, the volume fraction of texture components is very small.

4. The occurrence of strain hardening is well supported from the increase in hardness of the deformed sheets.

\section{Acknowledgment}

The authors acknowledge Defence Research and Development Organization (DRDO), India for providing financial support to the present work. We express our sincere thanks to the staff and students of Laboratory for Hypersonics and Shock Wave Research in the Department of Aerospace Engineering and to the students of Laboratory for Texture and Related Studies in Department of Materials Engineering at Indian Institute of Science, Bangalore for all the help during the study.

\section{References}

[1] J. Goodwin, Technol. Cult. 7 (1964) 268

[2] A. Rohatgi, A. Soulami, E.V. Stephens, R.W. Davies, M.T. Smith, J. Mater. Process. Technol. 214 (2014) 722.

[3] S.F. Golovashchenko, N.M. Bessonov, A.M. Ilinich, J. Mater. Process. Technol. 211 (2011) 875

[4] M. Stoffel, Mech. Res. Commun. 33 (2006) 775.

[5] M. Stoffel, Mech. Res. Commun. 33 (2006) 771.

[6] R. Shabadi, S. Suwas, S. Kumar, H.J. Roven, E.S. Dwarkadasa, Mater. Sci. Eng.: A 558 (2012) 439.

[7] R.J. De Angelis, J.B. Cohen, Trans. Am. Soc. Metals 58 (1965) 700.

[8] G.T. Higgins, Metall. Trans. 2 (1971) 1277.

[9] L.F. Trueb, J. Appl. Phys. 40 (1969) 2976.

[10] M.F. Rose, T.L. Berger, Philos. Mag. 17 (1968) 1121.

[11] A.G. Dhere, H. Kestenbach, M.A. Meyers, Mater. Sci. Eng. 54 (1982) 113.

[12] S. Suwas, A.K. Singh, K.N. Rao, T. Singh, Z. Metall. 93 (2002) 928.

[13] S. Suwas, A.K. Singh, Mater. Sci. Eng.: A 356 (2003) 368.

[14] N.P. Gurao, S. Sethuraman, S. Suwas, Mater. Sci. Eng.: A 528 (2011) 7739.

[15] M.B. Chen, J. Li, Y.M. Zhao, H. Yuan, W.C. Liu, Mater. Character. 62 (2011) 1188.

[16] J.J. Sidor, R.H. Petrov, L.A.I. Kestens, Mater. Character. 62 (2011) 228.

[17] S. Roy, S. Singh, S. Suwas, S. Kumar, K. Chattopadhyay, Mater. Sci. Eng.: A 528 (2011) 8469.

[18] G.E. Dieter, D. Bacon, Mechanical Metallurgy, McGraw-Hill, 1988. 